\title{
The Role of CD34 and D2-40 in the Differentiation of Dermatofibroma and Dermatofibrosarcoma Protuberans
}

\author{
Canan SADULLAHOĞLU', Yelda DERE², Türkan REZANKO ATASEVER³, Mine TUNAKAN ÖZTOP³, \\ Önder KARAASLAN ${ }^{4}$
}

Department of Pathology, 'Antalya Training and Research Hospital, ANTALYA, TURKEY, ${ }^{2}$ Mugla Sıtkı Koçman University Faculty of Medicine, MUGLA, TURKEY, ${ }^{3}$ Izmir Ataturk Training and Research Hospital, IZMIR, TURKEY,

${ }^{4}$ Department of Plastic and Reconstructive Surgery, Izmir Ataturk Training and Research Hospital, IZMIR, TURKEY

This study was presented as poster presentation at the $22^{\text {nd }}$ National Congress of Pathology, Antalya, Turkey, held between 7-11 November 2012 and $27^{\text {th }}$ European Congress of Pathology, Serbia, Belgrade, held between 5-9 September 2015.

\begin{abstract}
Objective: Dermatofibroma (DF) is a benign fibrohistiocytic tumor whereas dermatofibrosarcoma protuberans (DFSP) has intermediate malignant potential. CD34 is the most commonly used antibody in differentiating these tumors. Various studies have stated the rates of D2-40 expression as $0-50 \%$ in DFSPs and $86-100 \%$ in DFs. Our aim in this study was to determine the expression of CD34 and D2-40 in DFs and DFSPs and the possible use of D2-40 in the differential diagnosis of these lesions.
\end{abstract}

Material and Method: This is a retrospective study including 30 DF and 15 DFSP cases which were reevaluated for epidermal changes, the presence of a transmission zone (Grenz zone), infiltration of soft tissues, infiltration pattern and histologic subtypes in addition to cellular pleomorphism, nuclear atypia, and necrosis. A manual immunohistochemistry procedure was performed with D2-40 and CD 34 antibodies using a representative paraffin block.

Results: The average age was 37.36 and 42.86 years in the DF and DFSP cases. The average diameter was 0.9 and $5.03 \mathrm{~cm}$, respectively, for the DFs and DFSPs. There was a significant correlation between the two entities for sex, localization and diameter of the lesion. A significant difference was found between the positivity of CD34 and D2-40 in DFs and DFSPs.

Conclusion: Additional immunohistochemical markers may be needed in DFs with CD34 positivity. Our results showed the additional helpful role of this marker in problematic cases.

Key Words: Dermatofibroma, Dermatofibrosarcoma protuberans, CD34, D2-40, Podoplanin

\section{INTRODUCTION}

Dermatofibroma (DF) is a benign fibrohistiocytic tumor usually seen in women and located in the limbs. Dermatofibrosarcoma protuberans (DFSP) is a fibrohistiocytic tumor with intermediate malignant potential. The recurrence rate and the metastatic potential is higher in DFSP. Due to the differences in treatment and prognosis, it is important to diagnose these lesions correctly. In practice, CD34 and Factor XIIIa markers are often used to distinguish DP from DFSP $(1,2)$. Some studies have reported focal positivity with CD34 in DF, especially in the peripheral region of the lesion (3). D2-40, a monoclonal antibody usually known as podoplanin, is the main marker of lymphatic endothelium. Various studies have stated the rate of D2-40 expression as $0-50 \%$ for DFSPs and $86-100 \%$ for DFs (4-7). In this study, we aimed to determine the expression of CD34 and D2-40 in DFs and DFSPs together with the possible use of D2-40 as a second step marker after CD34 in differential diagnosis.

(Turk Patoloji Derg 2017, 33:223-227)

Received : 06.03.2017 Accepted : 04.05.2017

\section{MATERIAL and METHOD}

This study includes 30 DF and 15 DFSP cases diagnosed at Izmir Katip Çelebi University Ataturk Training and Research Hospital's Department of Pathology between 2005 and 2011. The study has ethical approval from the 9 Eylul University Ethics Committee (approval no: 2011/35-17).

We collected demographic data of the cases from the hospital records. The age and sex of the patients, the localization and diameter of the lesion, and the state of recurrence and metastasis were recorded. All the hematoxylin-eosin stained slides were revised and epidermal changes, the presence of a transmission zone (Grenz zone) between the epidermis and the lesion, the infiltration of perilesional soft tissues and the infiltration pattern, and the histological subtypes were noted in addition to the presence of cellular pleomorphism, nuclear atypia, and necrosis. A demonstrative paraffin-embedded block was selected for immunohistochemical study in all cases. A

Correspondence: Canan SADULLAHOĞLU

Antalya Training and Research Hospital, Department of Pathology,

ANTALYA, TURKEY

E-mail: canan-rana@hotmail.com Phone: +90 5366757325 
manual immunohistochemistry procedure was performed with D2-40 (Monoclonal Mouse Anti-human, clone D240, DAKO) and CD 34 (Monoclonal Mouse Anti-human, clone QBEnd 10, DAKO) antibodies. Internal vascular structures were accepted as positive control for CD34 and mesothelioma was used as the external positive control for D2-40. Cytoplasmic staining for CD34 and membranous and cytoplasmic staining for D2-40 were accepted as positive and the expressions were classified as follows: no staining was negative; $1-25 \%$ of the cells staining, including patchy and peripheral staining, was focal positive; and over $25 \%$ the cells staining was diffuse positive.

Statistical analysis was carried out with the SSPS 14.0 software (SPSS, Inc., Chicago, USA). The statistical associations between CD34 and D2-40 expression in the histological samples were assessed using the chi-squared test. Clinicopathological variables were assessed with the chi-squared and Fisher exact tests. A $p$ value of less than 0.05 was considered to be statistically significant.

\section{RESULTS}

The average of age in the DF and DFSP cases was 37.36 and 42.86 years, respectively. In the DF group, 22 (73.3\%) cases were female and 8 (26.7\%) were male. The male patients formed $60 \%$ of the DFSP cases with 9 cases. The localizations of the DF was the limbs in $70 \%$, the trunk in $23 \%$ and the head and neck region in $7 \%$ s. DFSPs were located on the trunk in $66.7 \%$, head and neck region in $20 \%$, and the limbs in $13.3 \%$. The average diameter of DFs and DFSPs were measured as 0.9 and $5.03 \mathrm{~cm}$, respectively. No significant relationship was found between the DFs and DFSPs according to age distribution $(\mathrm{p}=0.245)$. However, there was a significant correlation for sex, localization and diameter of the lesion between the two entities $(p=0.03$, $\mathrm{p}=0.002, \mathrm{p}=0.001$ ). Eleven of 15 DFSPs had follow-up in our study and only one of these had a lung metastasis in the two years after the diagnosis.

Epidermal hyperplasia and Grenz zone were more frequent in DFs whereas the infiltration of lipomatous tissue and mitosis were usually observed in DFSPs (Figure 1A,B). Among the DF cases, 6 could be subclassifed as the atrophic type in 3 , the cellular type in 2 and the lipidized type in 1 whereas 24 were unclassified. One of the 15 DFSP cases showed fibrosarcoma-like and 2 showed malignant fibrous histiocytoma-like areas. In addition 2 DFSPs were associated with myxoid changes.

Seven DF cases expressed CD34 only focally while diffuse CD34 expression was observed in 14 (93.3\%) of the DFSPs (Figure 1C). None of the DF cases showed diffuse expression of CD34. Focal positivity was mostly in the peripheral region or in central areas but patchy (Figure $2 \mathrm{~A}$ ). The atrophic type ( 3 cases), cellular type ( 2 cases) and lipidized type (1 case) had no CD34 expression. CD34 positivity was lower in the myxoid and sarcomatoid differentiated areas of DFSPs (Figure 2B).

Six of the 30 (20\%) DFs showed focal and 23 cases (76.7\%) showed diffuse D2-40 expression (Figure 2C). Of the 15 DFSPs, only 3 (20\%) showed focal and 2 (13.3\%) showed diffuse positivity with D2-40. A significant difference was found between the CD34 and D2-40 positivity of the DFs and DFSPs ( $\mathrm{p}=0.000, \mathrm{p}=0.000)$ (Table I).

\section{DISCUSSION}

Dermatofibroma (DF) and Dermatofibrosarcoma protuberans (DFSP) are fibrohistiocytic tumors with unknown histogenesis (1). DFs are generally seen in females with a limb localization and a mean diameter of $1 \mathrm{~cm}$ while DFSPs are more common in males with a trunk localization and a mean diameter of $5 \mathrm{~cm}(1,8,9)$. In our study, the mean age of DFs and DFSPs were not statistically significantly different and were concordant with the literature. DFs and DFSPs had a sex distribution, size and localization similar to that reported in the literature $(1,2)$. DFSP has a local recurrence rate of $7 \%$ after extensive resection with negative margins. Its metastasis rate is $5 \%$, most commonly to the regional lymph nodes and lung $(1,2)$. We followed-up 11 DFSP cases in this study and only one had lung metastasis in the two years after the diagnosis.

Table I: The positivity rate of CD34 and D2-40 in Dermatofibroma and Dermatofibrosarcoma Protuberans

\begin{tabular}{lcccc}
\hline & \multicolumn{2}{c}{ CD34 } & \multicolumn{2}{c}{ D2-40 } \\
\cline { 2 - 5 } & DF & DFSP & DF & DFSP \\
\hline Negative & $23(76.6 \%)$ & 0 & $1(3.3 \%)$ & $10(66.7 \%)$ \\
\hline Focal Positive & $7(23.4 \%)$ & $1(6.7 \%)$ & $6(20 \%)$ & $3(20 \%)$ \\
\hline Diffuse Positive & 0 & $14(93.3 \%)$ & $23(76.7 \%)$ & $2(13.3 \%)$ \\
\hline Total Positivity & $7(23.4 \%)$ & $15(100 \%)$ & $29(96.7 \%)$ & $5(33.3 \%)$ \\
\hline
\end{tabular}

DF: Dermatofibroma; DFSP: Dermatofibrosarcoma protuberans. 

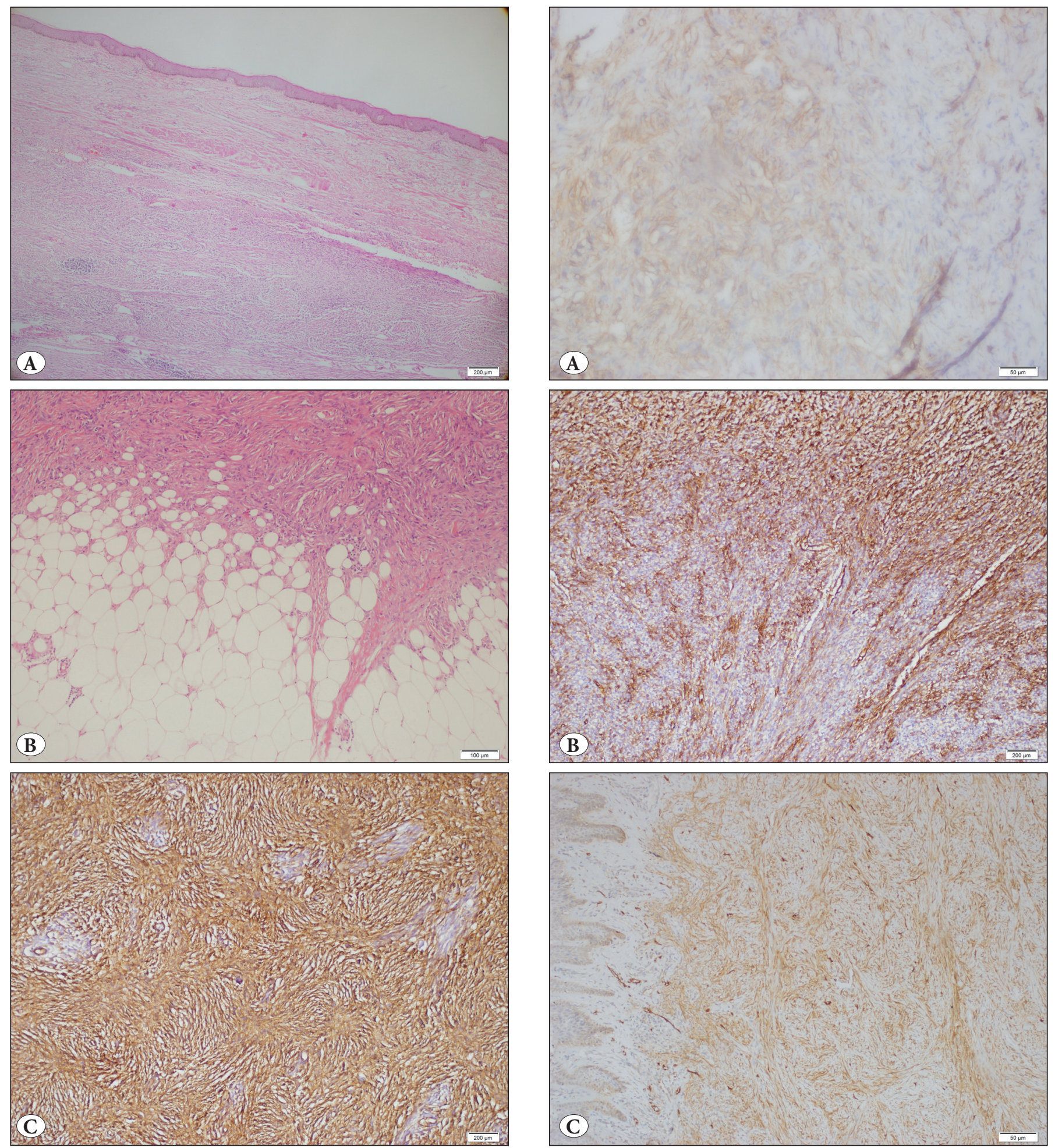

Figure 1: A) Grenz zone in Dermatofibroma, (H\&E; $\mathrm{x} 40$ ). B) Honeycomb infiltration of lipomatous tissue in Dermatofibrosarcoma Protuberans (H\&E; x200). C) Diffuse positivity of CD34 in Dermatofibrosarcoma Protuberans (CD34; $\mathrm{x} 200)$.

Figure 2: A) CD34 showed focal positivity in the peripheral region of Dermatofibroma (CD34; $\mathrm{x} 400)$, and B) in myxoid areas of Dermatofibroma Protuberans (CD34; x200). C) Diffuse positivity of D2-40 in Dermatofibroma (D2-40; x100). 
We also found that histopathologic features such as a Grenz zone and epidermal hyperplasia were more common in DFs, as reported by Korkolis et al. (10).

CD34 is a $115 \mathrm{KD}$ cell surface glycoprotein expressed by myeloid and lymphoid progenitors and endothelial cells $(8,11,12)$. In our study, CD34 positivity was observed in $23.4 \%$ of DFs and was only focally positive in the peripheral region of the lesion. However, 14 of the 15 DFSPs (93.3\%) showed diffuse CD34 positivity, as in the findings reported by Reimann and Fletcher (13) and Raica et al. (14). The focal positivity in one case could be related to the extensive myxoid change seen in that case.

D2-40 (podoplanin) is a monoclonal antibody of sialoglycoprotein and is used as a lymphatic endothelial marker in determining lymphatic invasion of solid tumors. Tumors with D2-40 expression include lymphangiomas and Kaposi sarcoma, ependymoma, meningioma, chondroid tumors and seminomas (14).

The results of studies on D2-40 expression in soft tissue tumors have shown that follicular dendritic cells and tumors originating from these cells express D2-40. However, there is limited data on the expression of D2-40 in DF and DFSPs (4-6). The more frequent expression of D2-40 in DFs in our study is concordant with reports suggesting a dermal dendrocytic origin of the lesion. In addition, D2-40 was expressed less in DFSPs, suggesting that the DFSP does not originate from dermal dendrocytes and does not contain cells epithelial-mesenchymal transition (6).

Bandarchi et al. have stated that all DFs showed D2-40 with $100 \%$ negativity in DFSPs and recommended D240 as a helpful marker in differentiating these two lesions (4). In addition, Kaddu and Leinweber suggested that D2-40 expression could be used in differentiating cellular neurothekeomas and DFs from similar entities in their study on 30 DFs and 15 cellular thekeomas (5). Our results also showed a statistically significant relationship between the D2-40 expressions of DFs and DFSPs. However, a controversial result from the $\mathrm{Xu}$ et al. study revealed that many malignant or benign subtypes of soft tissue tumors may show D2-40 expression with no statistically significant specificity (6).

In conclusion, DFs and DFSPs may show similar morphological and immunohistochemical features causing problems in the differential diagnosis, especially in cases without adequate sampling of the lesions. Additional immunohistochemical markers may be needed in DFs with CD34 positivity or DFSPs with myxoid degeneration and sarcomatous differentiation where the CD34 positivity decreases. Our results showed that the higher positivity of D2-40 in DFs compared to DFSPs indicates an additional helpful role for this marker in the differential diagnosis of problematic cases. Further studies are needed to prove the importance of D2-40 in fibrohistiocytic tumors and especially DFs because of the controversial results reported in the literature. In addition, more studies are needed to enlighten the histogenesis of these tumors which may be categorized by their origin in the future.

\section{ACKNOWLEDGEMENTS}

Thanks to Prof. Dr. Fulya Çakalağaoğlu and Dr. Neşe Ekinci for her contribution to the study and Hüseyin Candan for statistical analyses.

\section{CONFLICT of INTEREST}

The authors declare no conflict of interest.

\section{REFERENCES}

1. Weiss WS, Goldblum JR. Benign fibrohistiocytic tumors and fibrohistiocytic tumors of intermediate malignancy, In: Enzinger \& Weiss's soft tissue tumors. Philadelphia: Elsevier; 2008. 331402 .

2. Strutton G, Rubin AI, Weedon D. Tumors and tumor-like proliferations of fibrous and related tissues. In: Strutton G, Rubin AI, Weedon D, editors. Weedon's skin pathology. Philadelphia: Elsevier; 2011. 810-844.

3. Luzar B, Calonje E. Cutaneous fibrohistiocytic tumours- an update. Histopathology. 2010;56:148-65.

4. Bandarchi B, Ma L, Marginean C, Hafezi S, Zubovits J, Rasty G. D2-40, a novel immunohistochemical marker in differentiating dermatofibroma from dermatofibrosarcoma protuberans. Mod Pathol. 2010;23:434-8.

5. Kaddu S, Leinweber B. Podoplanin expression in fibrous histiocytomas and cellular neurothekeomas. Am J Dermatopathol. 2009;31:137-9.

6. Xu Y, Agose A, Kawashima $\mathrm{H}$, et al. High-level expression of podoplanin in benign and malignant soft tissue tumors; Immunohistochemical and quantitative real-time RT-PCR analysis. Oncol Rep. 2011;25:599-607.

7. Plaza JA, Ortega PF, Stockman DL, Suster S. Value of P63 and podoplanin (D2-40) immunoreactivity in the distinction between primary cutaneous tumors and adenocarcinomas metastatic to the skin: A clinicopathologic and immunohistochemical study of 79 cases. J Cutan Pathol. 2010;37:403-10.

8. Han TY, Chang HS, Lee JHK et al. A clinical and histopathological study of 122 cases dermatofibroma (Benign Fibrous Histiocytoma). Ann Dermatol. 2011;23(2):185-91.

9. Sanmartin O, Llombart B, López-Guerrero JA, Serra C, Requena C, Guillén C. Dermatofibrosarcoma protuberans. Actas Dermosifiliogr. 2007;98:77-87.

10. Korkolis DP1, Liapakis IE, Vassilopoulos PP. Dermatofibrosarcoma protuberans: Clinicopathological aspects of an unusual cutaneous tumor. Anticancer Res. 2007;27:1631-4. 
11. Tardio JC. CD34-reactive tumors of the skin. An updated review of an ever-growing list of lesions. J Cutan Pathol. 2008;36:89-102.

12. Li N, McNiff J, Hui P. Differential expression of HMGA1 and HMGA2 in dermatofibroma and dermatofibrosarcoma protuberans: Potential diagnostic applications, and comparison with histologic findings, CD34, and Factor XIIIa immunoreactivity. Am J Dermatopathol. 2004;26:267-72.
13. Reimann JD, Fletcher CDM. Myxoid dermatofibrosarcoma protuberans: A rare variant analyzed in a series of 23 cases. Am J Surg Pathol. 2007;31:1371-77.

14. Raica M, Cimpean AM, Ribatti D. The role of podoplanin in tumor progression and metastasis. Anticancer Res. 2008;28: 2997-3006. 